\title{
Sialolithiasis leading to sialadenitis- A case report
}

\author{
Muhaseena Min , Rahul Tiwari ${ }^{2, *}$, Heena Tiwari ${ }^{3}$, Abhishek Jairaj ${ }^{4}$, Arun Ramaiah ${ }^{5}$ \\ ${ }^{1}$ Lecturer, Dept. of Biomedical Dental Sciences, College of Dentistry, Imam Abdulrahman Bin Faisal University, Dammam, \\ Kingdom of Saudi Arabia, ${ }^{2}$ FOGS, MDS, Dept. of OMFS \& Dentistry, JMMCH \& RI, Thrissur, Kerala, ${ }^{3}$ BDS, PGDHHM, \\ Government Dental Surgeon, CHC Makdi, Kondagaon, Chhattisgarh, ${ }^{4}$ Senior Lecturer, Faculty of Dentistry, AIMST University, \\ Semeling Kedah Malaysia, ${ }^{5}$ Senior Fellow, Cleft \& Craniofacial Centre, St. Thomas Hospital, Malakkara, Pathanamthitta, \\ Chengannur, Kerala, India
}

\section{*Corresponding Author: Rahul Tiwari}

Email: drrahulvctiwari@gmail.com

\begin{abstract}
Sialolithiasis is the obstruction of salivary gland due to blockage of its excretory duct in response to the formation of calcareous concretions which causes the dilation of salivary gland. Sialadenitis is an acute infection of salivary gland. Sialadenitis is seen mostly in parotid gland and sialolith is mostly reported in sub-mandibular gland. We hereby report a case of elderly female with an acute pain on left sub-mandibular region which was diagnosed as sub-mandibular sialolithiasis leading to sialadenitis.
\end{abstract}

Keywords: Sialolithiasis, Sialadenitis, Calculi, Conservative management.

\section{Introduction}

The microbiology of sialadenitis is rarely reported in sub-mandibular \&sub-lingual gland. ${ }^{1}$ Staphylococcus, streptococcus \& gram-negative bacteria are the etiologic factors. Sialolith or salivary gland calculi are reported as 12 in 1000 adult population with double prevalence in males. It belongs to category of idiopathic dystrophic calcification. ${ }^{2}$ Foreign bodies, bacterial colonies, mucal plugs, cell remnants shed ductal epithelial cells cause the mineralization of calculi. ${ }^{3} 80 \%$ of sialolith occur in submandibular, $2 \%$ in sub-lingual, $6 \%$ in parotid gland \& $1 \%$ in minor salivary gland. The reason is its length and diameter, alkaline $\mathrm{pH}$ and salivary flow against the gravity. ${ }^{4}$ (Fig. 1) Sialolith are round to ovoid in shape, rough or smooth in texture \& yellowish in colour. ${ }^{5}$ Diagnosis is done by clinical examination, conventional radiography, ultrasound (USG) \& higher imaging technique like computed tomography (CT) scan. Sialography is also a recommended method of evaluation. ${ }^{6}$ Small sialolith which are located in periphery can be removed with gentle manipulation but surgical excision of gland is preferred. ${ }^{7}$ Treatment of sialadenitis is rehydration of the patient, sialagogues, using lemon drops to initiate the salivary flow, massaging gland and treating with antibiotics. If there is suppuration, it needs surgical drainage. ${ }^{8}$

\section{Case Report}

A 32-year-old female presented with a complaint of pain in the left lower back region of jaw for 2 days and also a feeling of some mass in the cervical region on the left side. History of pain was sudden in onset with moderate intensity \& continuous nature. Patient revealed that pain was aggravating while taking food \& it is also radiating towards the left ear since last. Patient was having mild rise in temperature for 12 hours. Patient gives no relevant history of increase or decrease size of the present mass. On extraoral examination no facial asymmetry was present. On palpation of left submandibular gland there was enlargement \& a palpable $1 * 1 \mathrm{~cm}$ in size roughly oval in shape, firm, tender\& mobile node present. There was no difficulty in mouth opening \& closing and normal jaw movements. Intraoral examination revealed a carious tooth in response to mandibular left $3^{\text {rd }}$ molar. The tooth was non-tender on percussion \& no signs of localized infection or inflammation were present. An intraoral periapical radiograph of $3^{\text {rd }}$ molar confirmed the clinical diagnosis. As patient was complaining of earache, an ENT consultation was taken after which we planned to take ultrasonography of the submandibular gland. Ultrasonography of the neck revealed left submandibular gland enlarged and was having coarsened echo texture with increased vascularity. Duct appeared dilated with a hyperechoic focus of $2.2 \mathrm{~cm}$ noted within. Few adjacent enlarged submandibular lymph nodes noted largest measuring $13 \times 6 \mathrm{~mm}$ with preserved fatty hilum. No foci of infection, abscess or collection was present. Ultrasonography impression confirmed evidence of left sub mandibular sialolithiasis and sialadenitis. (Fig. 2) Patient was treated with aerobic, anaerobic and gram-negative antibiotics with analgesics for five days. Patient was advised to take salt water gargles quarterly in a day for five days. Patient was kept under regular follow up and her symptoms were released after seven days. Carious tooth was removed on further follow up. 


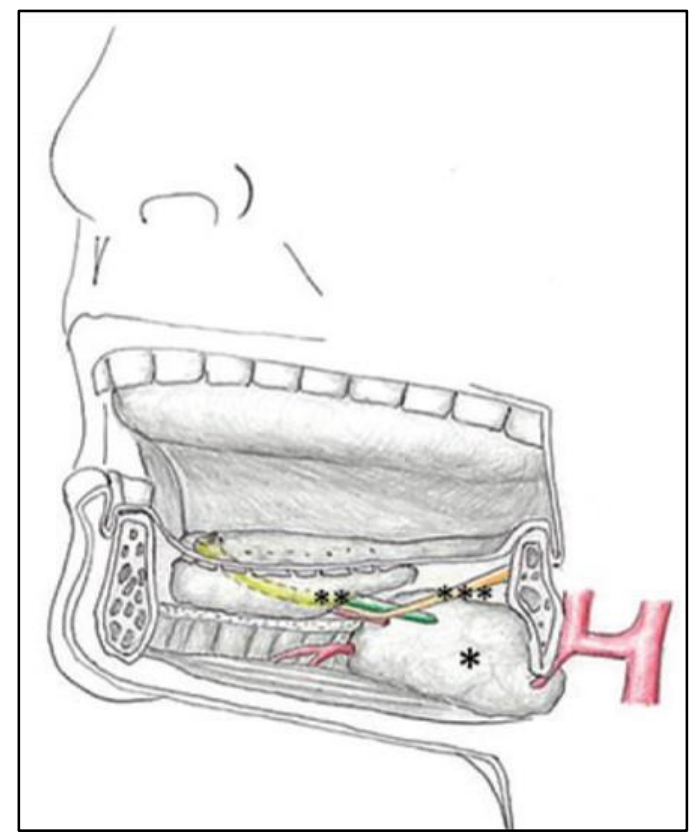

Fig. 1: Anatomy of submandibular gland

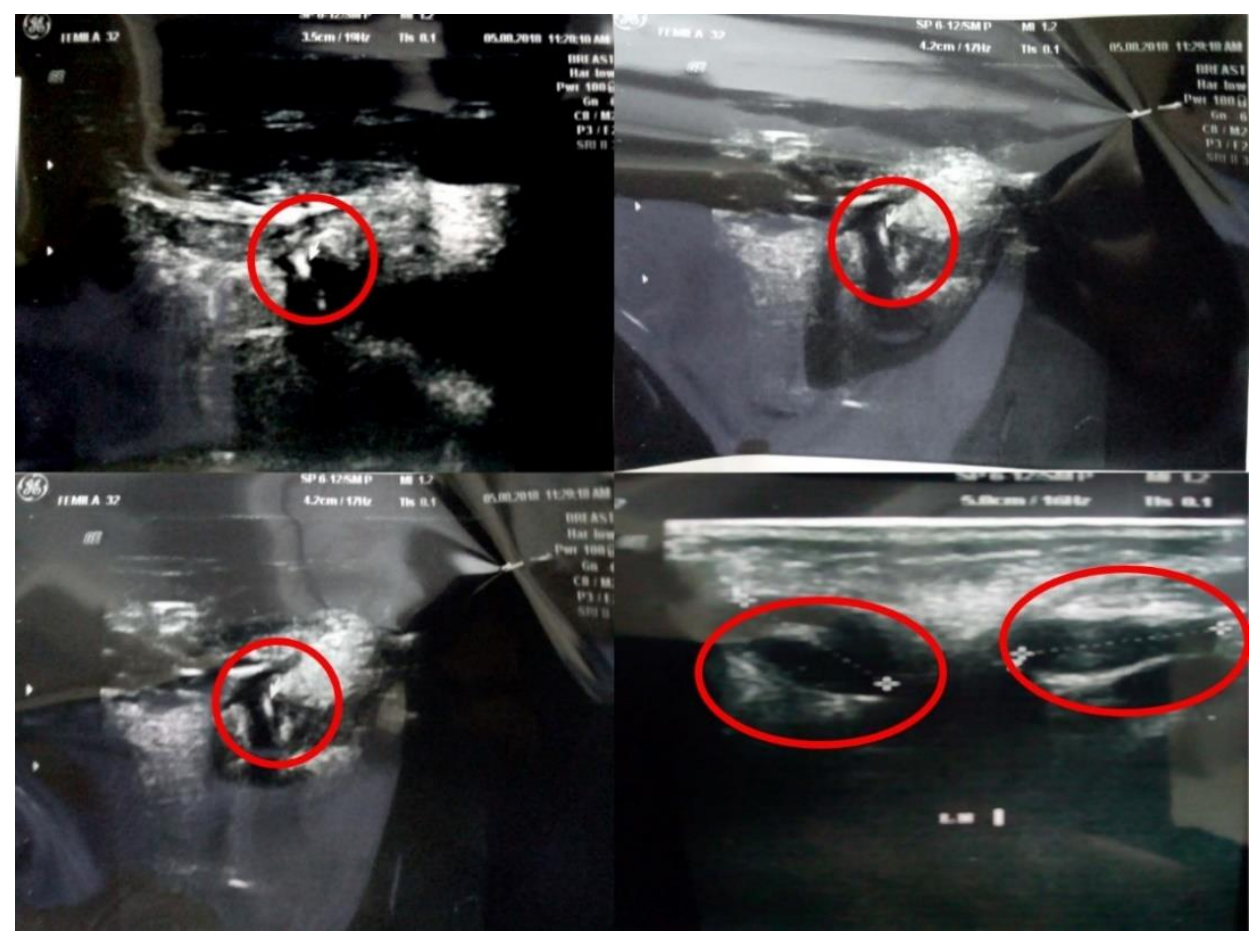

\section{Fig. 2: USG showing sialolith lesion}

\section{Discussion}

Sialadenitis is the inflammation of salivary gland. This can happen due to various etiologic factors in which poor oral hygiene and dehydration are more prone to cause. It is acute and chronic. In untreated cases it can lead to formation of mass. Sialolithiasis is the formation of salivary calculi which causes the blockage of salivary duct minimizing the salivary flow. Repeated episodes of acute infection and retrograde infection by low grade opportunistic oral flora can result directly in chronic recurrent sialadenitis. This result in increased mucous content of secretions, stasis and further episodes of inflammation. Usually there is ductal obstruction, associated with stones and structure of the main duct system. The other causes include mucous plugs, injury to the duct and papilla, and ductal compression by a tumor. Sometimes the cause may remain uncertain..$^{9}$ The effect of radiation results in a permanently reduced salivary flow thus predisposing the gland to an ascending infection. ${ }^{10}$ Sometimes it is impossible to identify a cause. Histologically loss of 
acini, duct dilatation and a scattered chronic inflammatory cellular infiltrate and calculi are seen. Ultrasound, CT and MRI scans are preferred diagnostic tools. Sialagogues, frequent gland massages and antibiotics for acute exacerbations are conservative approach of treatment and in extensive cases surgical excision of gland is done.

\section{Conclusion}

Sialolithiasis and sialadenitis can be managed conservatively in acute phases but in chronic case it can lead to long term complications which needs substantive treatment planning. Giant lesions mostly require surgical removal. Prompt diagnosis and treatment are the keys to achieve better outcome for increasing quality of life of patient. This case report illustrates a case of submandibular sialolithiasis leading to sialadenitis that was diagnosed clinically and radiographically and treated conservatively with no complications.

\section{References}

1. McQuone SJ. Acute viral and bacterial infections of the salivary glands. Otolaryngol Clin North Am. 1999;32:793-811.
2. Chandra Mouli Sialolith: A Case Report with Review of Literature. Indian Journal of Multidisciplinary Dentistry. 2012;(1):2.

3. Liao G, Su Y. Sialoendoscopic secondary intervention after failure of open sialolithectomy. J Oral Maxillofac Surg. 2010;68(2):313-318.

4. Raksin SZ, Gould SM, William AC. Submandibular gland sialolith of unusual size and shape. Journal of Oral Surgery. 1975;33:142-145.

5. Zenk J, Benzel W, Iro H. New modalities in the management of human sialolithiasis. Minimally Invasive Therapy \& Allied Technologies. 1994;3:275-284.

6. Martin S. Greenberg, Burket's oral medicine, 11th edi: ch 8; pg 191-223.

7. Laliytha Kumar Bijai, Venkatesh Jayaraman, and Ravi David Austin, "Chronic Bacterial Sialadenitis-A Case Report." Oral Surgery, Oral Medicine, Oral Radiology. 1, no. 1 (2013):1-5. doi: 10.12691/oral-1-1-1.

8. Bhatty MA, Piggot TA, Soames JV. Chronic non-specific parotid sialadenitis. Br J Plast Surg. 1998;51:517-521.

9. Sharer WG, Hine MK, Levy BM. A Textbook of Oral Pathology. Philadelphia: WB Saunders Company, 1983.

10. Seifert WG, Waller D. Klassifikation der Parotiszysten. Differential diagnose der Speichelgangzysten und lymphoepithelialen Zysten. Laryngol Rhinol Otol. 1982;61:78 86 . 